\title{
Blutdruckgrenzwerte beim systemischen Lupus erythematodes
}

Tselios K et al. Impact of the new American

College of Cardiology/American Heart Association definition of hypertension on atherosclerotic vascular events in systemic lupus erythematosus. Ann Rheum Dis 2020; 79: 612-617. doi: 10.1136/ annrheumdis-2019-216764

Vor einigen Jahren definierten das American College of Cardiology und die American Heart Association die Hypertonie neu: Ein Bluthochdruck liegt nun gemäß ihrer Leitlinie bereits bei systolischen bzw. diastolischen Werten von 130 bzw. $80 \mathrm{mmHg}$ oder mehr vor. Angesichts des erhöhten kardiovaskulären Risikos beim systemischen Lupus erythematodes (SLE) stellt sich die Frage, ob diese neuen Grenzwerte auch für SLE-Patienten gelten sollten.

Bei der Grenzwert-Neudefinition wurden keine Patienten mit systemischen Autoimmunerkrankungen berücksichtigt und dem entsprechend fehlen Behandlungsempfehlungen für diese Kollektive, berichten kanadische Wissenschaftler. Um zu klären, wie gut sich anhand der neuen Hypertoniedefinition Atherosklerose-bedingte Komplikationen beim SLE vorhersagen lassen bzw. ob bei einem Schwellenwert von 130-139/80-89mmHg das kardiovaskuläre Risiko überproportional zunimmt, werteten sie die Daten von 1532 SLE-Patienten aus, die seit mindestens 2 Jahren an der University of Toronto Lupus Clinic betreut wurden und initial keine Atherosklerosekomplikationen aufwiesen. Gemäß ihrer durchschnittlichen Blutdruckwerte in diesen 2 Jahren stuften die Forscher die Patienten - unabhängig von einer begleitenden antihypertensiven Medikation - folgendermaßen ein:

- Normotensiv: Adjustierter durchschnittlicher Blutdruck $<130 / 80 \mathrm{mmHg}$

- Hypertonie Grad 1: Adjustierter durchschnittlicher Blutdruck 130139/80-89 mmHg

- Hypertonie Grad 2: Adjustierter durchschnittlicher Blutdruck $\geq$ $140 / 90 \mathrm{mmHg}$. 
Anschließend prüften die Wissenschaftler, wie viele Studienpatienten im Verlauf der weiteren Nachbeobachtungszeit Atherosklerose-bedingte Gefäßereignisse erlitten. Diese umfassten die neu aufgetretene Angina pectoris, den Myokardinfarkt, Revaskularisierungseingriffe, die Herzinsuffizienz auf der Basis einer Ischämie, die periphere Gefäßerkrankung mit Angioplastie, transiente ischämische Attacken (TIAs), den Schlaganfall sowie den Tod aufgrund eines Myokardinfarkts oder Schlaganfalls.

\section{Ergebnisse}

Die Studienpatienten (88,1\% Frauen) waren im Schnitt 36 Jahre alt und litten durchschnittlich seit 6 Jahren an der Erkrankung. 1061 Patienten (69,3\%) stuften die Forscher als normotensiv ein, 316 (20,6\%) hatten durchschnittliche Blutdruckwerte von $130-139 / 80-89 \mathrm{mmHg}$ und 155 $(10,1 \%)$ von $\geq 140 / 90 \mathrm{mmHg}$. Nach einer durchschnittlichen Nachbeobachtungszeit von 10,8 Jahren bzw. 16601 Patientenjahren verzeichneten die Studieninitiatoren insgesamt 124 Atherosklerose-bedingte Gefäßereignisse inklusive 20 Todesfälle. Die Prävalenz betrug dabei im Kollektiv der normotensiven Patienten bzw. der Grad 1- und Grad 2-Hypertoniker 4,8, 13 bzw. 20,6\% und die Inzidenzrate 4,5, 11,5 bzw. 18,9 pro 1000 Patientenjahren. Statistische Signifikanz bestand dabei sowohl für den Vergleich normotensiv/Grad 1-Hypertonie als auch für den Vergleich normotensiv/Grad 2-Hypertonie und den Vergleich Grad1-/ Grad 2-Hypertonie. Mittels multivariater Analyse (unter Berücksichtigung aller traditionellen und erkrankungsspezifischen Atherosklerose-Risikofaktoren) identifizierten die Wissenschaftler durchschnittliche Blutdruckwerte von 130-139/80-89 mmHg in den ersten 2 Beobachtungsjahren als unabhängigen Risikofaktor für spätere Atherosklerose-bedingte Gefäßereignisse (Hazard Ratio 1,73; $95 \%$ KI 1,13-2,69).Weitere Risikofaktoren stellten das Rauchen, die Behandlung mit Glukokortikoiden, die Behandlung mit Plättchenaggregationshemmern/Antikoagulanzien sowie die SLE-Krankheitsaktivität dar.

\section{FAZIT}

SLE-Patienten, die über einen Zeitraum von 2 Jahren durchschnittliche Blutdruckwerte von 139/80$89 \mathrm{mmHg}$ aufweisen, entwickeln im Vergleich zu normotensiven Patienten signifikant häufiger kardiovaskuläre Komplikationen, schlussfolgern die Autoren.

Angesichts dieser Studienergebnisse empfehlen sie, eine antihypertensive Therapie beim SLE frühzeitig einzuleiten und hierbei einen Blutdruckgrenzwert von $<130 / 80$ $\mathrm{mmHg}$ anzustreben.

Dr. med. Judith Lorenz, Künzell 